\title{
Pine Forest Height Inversion Using Single-Pass X-Band PolInSAR Data
}

\author{
Franck Garestier, Pascale C. Dubois-Fernandez, Senior Member, IEEE, \\ and Konstantinos Panagiotis Papathanassiou, Senior Member, IEEE
}

\begin{abstract}
A sparse pine forest is investigated at X-band on a single-pass polarimetric synthetic aperture radar interferometry (PolInSAR) data set using HH and HV channels. These first preliminary results show that the associated phase centers present a significant vertical separation (about $6 \mathrm{~m}$ ) allowed by penetration through gaps in the canopy. Forest parameter inversion using the random volume over ground (RVoG) model is evaluated and adapted at this frequency. The forest height can be retrieved accurately by supposing a high mean extinction coefficient (around $1.6 \mathrm{~dB} / \mathrm{m}$ ). The penetration depth is estimated to be around $4 \mathrm{~m}$, based on the forest height ground measurements. Finally, a time-frequency analysis using a sublook decomposition is performed to increase the vertical separation of the polarimetric phase centers. As a consequence, RVoG-inversion performance is improved, and a penetration depth that is in better accordance with a previous work (of the order of $2 \mathrm{~m}$ ) is found. This paper has shown that the height inversion of a pine forest was possible using PolInSAR X-band data and that the performance was more dependent on the forest density than at lower frequencies.
\end{abstract}

Index Terms-Forest, interferometry, polarimetric synthetic aperture radar interferometry (PolInSAR), polarimetry, X-band.

\section{INTRODUCTION}

C LIMATE understanding is a challenge for a wide community of scientists, which is currently addressed by modeling the Earth as a global system involving different cycles among which the carbon cycle is essential. One of the most dynamic carbon storages associated with land-atmosphere transfer is constituted by the forests for which the above-ground biomass is representative of the organic carbon quantity present in vegetation. Polarimetric synthetic aperture radar (SAR) interferometry (PolInSAR) is a new technique which has shown a good potential for forest height retrieval using the random volume over ground (RVoG) model [1], [2]. This model has been validated at L-band over different forests and terrain conditions. Forest height is an important parameter because it

Manuscript received April 27, 2007; revised May 6, 2007. This work was supported in part by the Program National de Télédétection Spatiale (PNTS).

F. Garestier and P. C. Dubois-Fernandez are with the Department of Electromagnetism and Radar/Radar IMageurs, Office National d'Études et de Recherches Aérospatiales, 13661 Salon-de-Provence, France (e-mail: fgarestier@yahoo.com).

K. P. Papathanassiou is with the Polarimetric Synthetic Aperture Radar Interferometry Research Group, Microwaves and Radar Institute, German Aerospace Center, 82234 Wessling, Germany.

Color versions of one or more of the figures in this paper are available online at http://ieeexplore.ieee.org.

Digital Object Identifier 10.1109/TGRS.2007.907602 can be linked to the above-ground biomass through allometric equations [3]. In this paper, the RVoG model [1], [2] is applied on a pine forest at X-band. A methodology adapted to the foliage-wave interaction specificity at this frequency is proposed for extracting the forest parameters.

The X-band single-pass polarimetric interferometric data acquired by RAMSES [4], constituting the support of this paper, are first briefly presented in Section II.

The analysis of the experimental data has shown an unexpected behavior in two of the four polarimetric channels. A system-induced decorrelation has corrupted the VH and VV channels along the whole scene. Since a loss of coherence in one polarimetric channel would bias the parameter extraction, only two polarizations are used for the inversion using the RVoG model, namely, HH and HV. Section III reviews the RVoG model assumptions and describes how to deal with only two polarizations for the extraction of the pine forest parameters [5].

Section IV is dedicated to data investigation. A significant difference between interferometric heights associated with the $\mathrm{HH}$ and $\mathrm{HV}$ channels is observed over the whole forest, indicating that the apparent penetration depth, i.e., the height difference between the top of the canopy and the phase-center heights, varies widely with the polarization state. The standard RVoG model is then adapted to the X-band wave interaction with the forest canopy, and the performance of inversion is evaluated in comparison with the ground measurements.

In Section V, a time-frequency analysis (TFA) is performed in order to increase the performance of the RVoG model inversion. Indeed, azimuth sublook decomposition, which is performed by filtering SAR image frequency spectra in this direction, allows better isolation of pure ground and canopy contributions in the HH and HV channels, respectively. The resulting penetration depth obtained with this optimization scheme is smaller than the one obtained with the full-resolution data and is better compared with the previous work results [6]. Finally, the accuracy of the parameter extraction (forest height retrieval) with the RVoG associated with TFA is evaluated.

\section{Description OF THE DATASET}

The Radar Imagery Thematiques Agricoles et Sols (RITAS) campaign was conducted in March 2002, and the data were acquired over the Institut National de Recherche Agronomique (INRA) test site of Avignon, France, by the experimental radar 

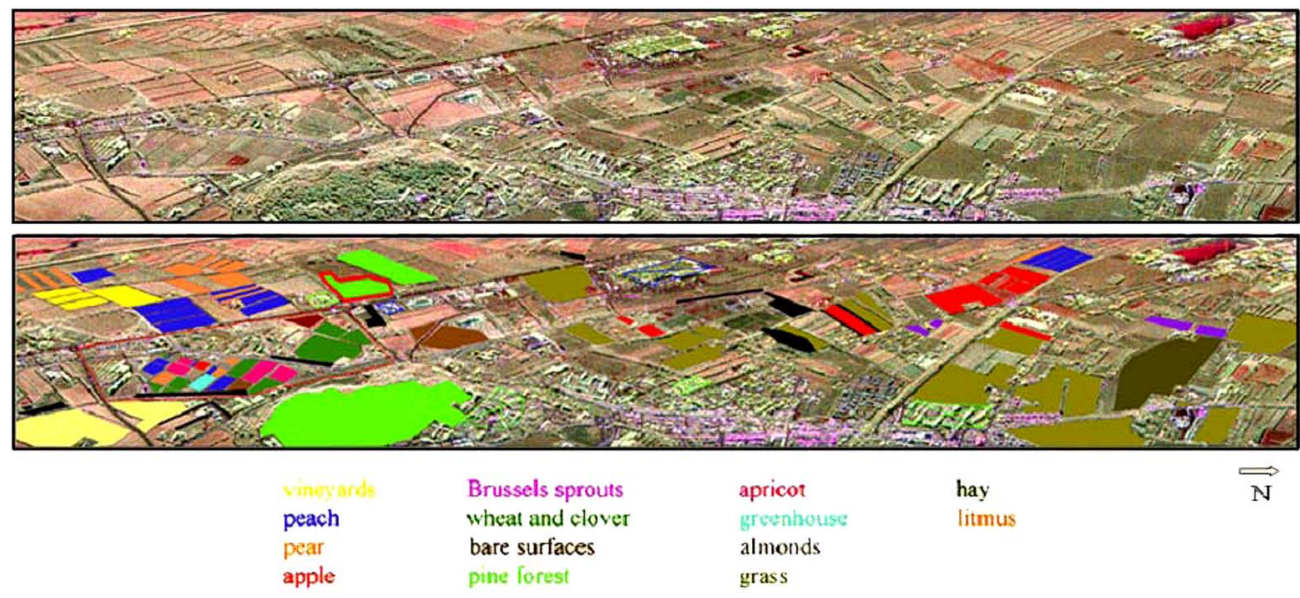

Fig. 1. Pauli-basis normalized polarimetric $\sigma_{0}$ map of the RITAS data. (Top) RGB coding: Red color corresponds to the intensity of HH+VV backscattering, green to $\mathrm{HV}$, and blue to HH-VV and (bottom) the description of the available ground truth. The studied pine forest is outlined in red.
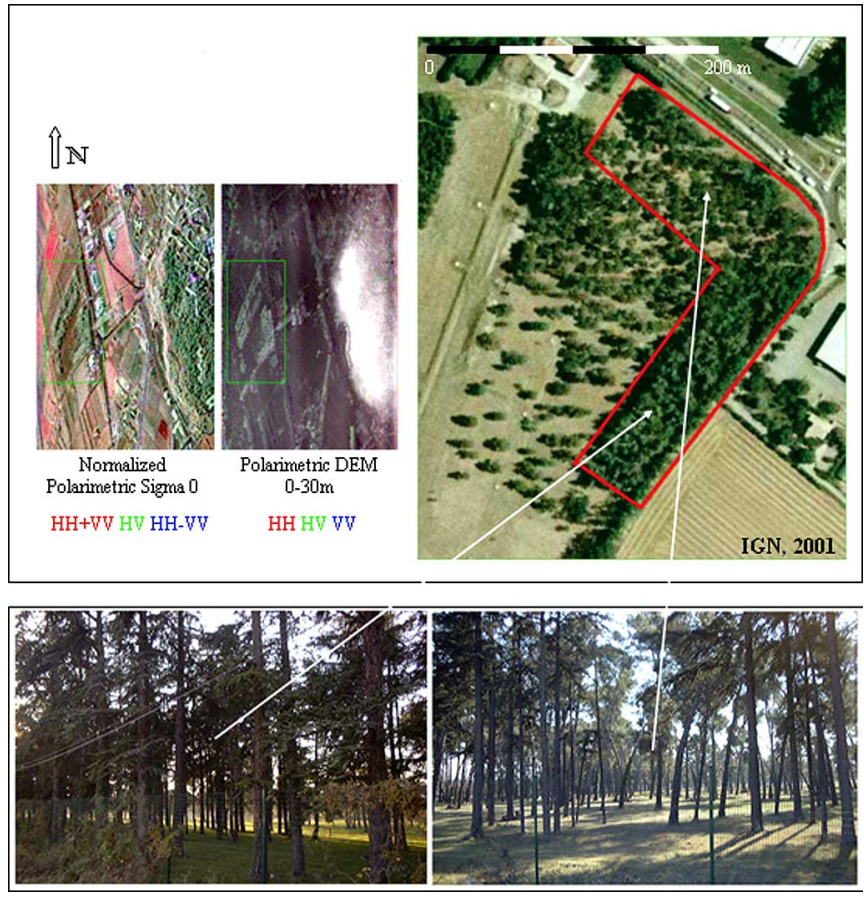

Fig. 2. Aerial photography and views of the studied pine forest. In this paper, only the red outlined zone is considered.

system RAMSES [4]. The test area is composed by orchards, bare fields, forests, vegetable patches, and a part of the village of Montfavet.

Fig. 1 shows the data set as a color composite where the backscattered power corresponding to the three Pauli-basis polarizations $(\mathrm{HH}+\mathrm{VV}, \mathrm{HV}, \mathrm{HH}-\mathrm{VV})$ is coded in $\mathrm{RGB}$ and normalized to improve the image dynamic. Bare fields appear in brown color, vegetation in green, and buildings, which are characterized by an important double-bounce scattering, in blue. Several pine forest stands are present in the scene, as shown in Fig. 1, and the study presented in this paper will concentrate on this type of forest. The largest pine forest patch has not been taken into account in this paper because of a strong underlying topography and the presence of man-made

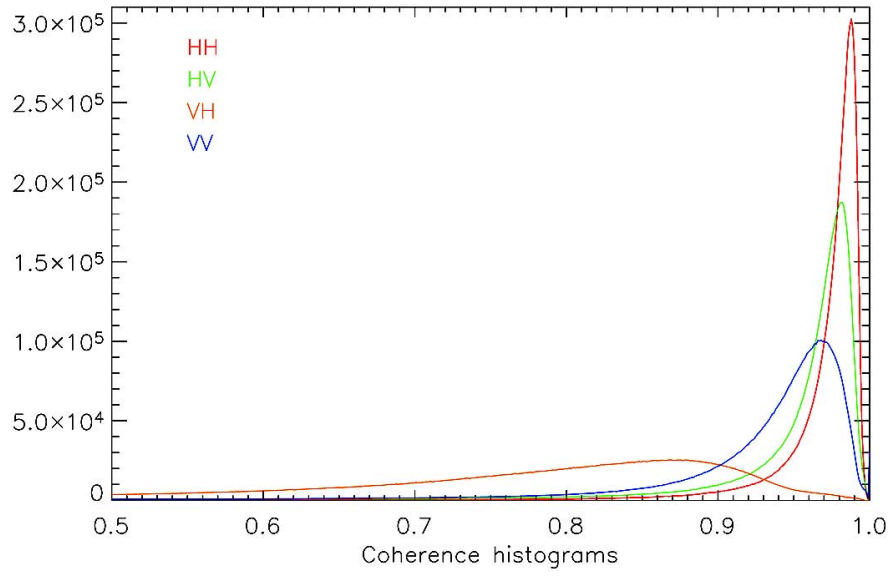

Fig. 3. Coherence histograms computed over the whole RITAS data set associated with the four polarimetric channels $\mathrm{HH}, \mathrm{HV}, \mathrm{VH}$, and VV.

structures under the canopy. Only one forest area was covered by the ground-truth campaigns and is considered in this paper (surrounded in red in Fig. 1).

A zoom of optical airborne photography, acquired the year before the X-band-data acquisition, is shown in Fig. 2 to provide some information on the tree density. It shows that the pine forest is relatively sparse and presents gaps in the canopy. This photography is juxtaposed to a normalized polarimetric $\sigma_{0}$ map expressed in the Pauli basis, where the mean values of $\sigma_{0}$ corresponding to these three polarization states have been shifted to a common one for a better dynamic, and to a polarimetric Digital Elevation Model (DEM) associated with the linear polarization basis, the color intensity being related to the $0-30-\mathrm{m}$ phase-center-height interval. On the polarimetric DEM, the brightest colors correspond to elevated scatterers (pine forest and hedges in green and a forested hill in white). The last two photographs correspond to different tree density areas. We can observe that the underlying ground is a flat surface covered by short grass with no understory. The height of this managed forest is homogeneous. The measurements made with 1-m accuracy range from 17 to $20 \mathrm{~m}$ ( $18 \mathrm{~m}$ in mean).

The boresight incidence angle of the acquisition is $30^{\circ}$, and the resolution is $0.9 \mathrm{~m}$ in range and in azimuth directions. 
Three trihedral corner reflectors were deployed on the scene for radiometric and polarimetric calibrations. One dihedral corner reflector was set with a $22.5^{\circ}$ tilt to provide a strong signal in all the four polarimetric channels; this target is useful to properly coregister the polarimetric channels. Finally, one trihedral corner reflector that is positioned on a 5-m mast was used for the interferometric calibration. A specific PolInSARcalibration procedure adapted to the deramp-on-receive mode [7] has been applied.

\section{Dual-Polarimetric (Dual-Pol) INVERSION WITH RVOG MODEL}

Due to a technical problem during the acquisition, the forest parameter inversion has been performed using only two polarimetric channels. This section begins with a quality investigation of the interferometric pairs associated with the linear polarization channels and the phase and amplitude of the interferometric coherence and establishes the inversion procedure for a Dual-Pol configuration.

Since these data have been acquired in a single-pass mode, the distance between antennas, i.e., the baseline, is constant, with its length equal to $63 \mathrm{~cm}$. As a direct result, the height of ambiguity (i.e., the height creating a $2 \pi$ phase shift) is large $(\simeq 250 \mathrm{~m}$ ), inducing a low height sensitivity. The accuracy associated with the PolInSAR calibration procedure [7] is estimated to be around $3^{\circ}$ and translates into a $2-\mathrm{m}$ vertical accuracy.

Coherence values are observed to be greater than 0.9 on natural surfaces and even around 0.98 for bare surfaces in the three channels. Fig. 3 shows the coherence histograms computed over the whole data set associated with the four polarimetric channels $\mathrm{HH}, \mathrm{HV}, \mathrm{VH}$, and VV. The VH coherence is affected by a strong decorrelation, and the one associated with VV is unexpectedly lower than the HV one. The noticeable decorrelations affecting the $\mathrm{VV}$ and $\mathrm{VH}$ polarizations are due to a perturbation in the $\mathrm{V}$ emission channel during the flight, where the high-power polarization switch was occasionally underperforming. In consequence, the PolInSAR inversion using the RVoG model [1], [2] was conducted on only two polarimetric channels, the $\mathrm{HH}$ and HV. The RVoG model inversion relies on the interferometric coherences computed for all the possible polarization states. As the inversion methodology uses the linear regression of interferometric coherences in the complex plane, a VV decorrelation would induce a bias in this inversion resulting to an overestimation of the forest height. Papathanassiou and Cloude [5] have shown that forest height and mean extinction coefficient inversions were possible at L-band with only two polarization channels with an associated small performance degradation over the full polar inversion and that the best Dual-Pol configuration is then the polarization couple $\mathrm{HH}$ and $\mathrm{HV}$.

The performance of the standard RVoG model inversion [1], [2] has been evaluated at L-band in the context of different forests and terrain conditions. This model assumes the vegetation to be a homogeneous layer of randomly oriented particles, which provides the same extinction for all the polarizations, and the ground surface to be an impenetrable scatterer. The complex interferometric coherence $\gamma(\mathbf{w})$ corresponding to one polarization state $\mathbf{w}$ is defined as a coherent combination of volume and surface scattering

$$
\begin{aligned}
\gamma(\mathbf{w}) & =\frac{\gamma_{v}+\mu(\mathbf{w})}{1+\mu(\mathbf{w})} e^{j \phi_{0}} \\
& =\left[\gamma_{v}+L(\mathbf{w})\left(1-\gamma_{v}\right)\right] e^{j \phi_{0}} \\
L(\mathbf{w}) & =\frac{\mu(\mathbf{w})}{1+\mu(\mathbf{w})}, \quad \text { with } 0 \leq L(\mathbf{w}) \leq 1 .
\end{aligned}
$$

$\phi_{0}$ denotes the ground interferometric phase under the assumption of no-ground decorrelation (the ground coherence is equal to one), and its variations over a forest are then directly related to the underlying topography. $\gamma_{v}$ is the complex coherence of the homogeneous random volume, and $\mu(\mathbf{w})$ represents the ground-to-volume power ratio, varying with polarization. For a given geometric configuration, $\gamma_{v}$ is the function of the following two forest parameters: the extinction coefficient $\sigma$, which corresponds to the mean extinction of the vegetation layer, the forest height $h_{v}$ and of the system parameters, the vertical wavenumber $k_{z}$, and the incidence angle $\theta$ [8], [9]

$$
\gamma_{v}=\frac{\int_{0}^{h_{v}} e^{\frac{2 \sigma z}{\cos \theta}} e^{j k_{z} z} d z}{\int_{0}^{h_{v}} e^{\frac{2 \sigma z}{\cos \theta}} d z} .
$$

For a given interferometric configuration, the volume decorrelation increases with increasing volume height and decreasing mean extinction coefficient.

The vertical wavenumber of the interferometric system is a function of the incidence angle difference between the master and the slave antenna $\delta \theta$, related to interferometer configuration and acquisition geometry, and the signal wavelength $\lambda$

$$
k_{z}=\frac{4 \pi}{\lambda \sin \theta} \delta \theta .
$$

The two forest physical parameters retrieved with the RVoG model are the forest height and the vegetation layer mean extinction coefficient. The inversion procedure described in [2] consists of the following steps: 1) the linear regression of the complex coherence associated with all the given polarization states in the complex plane; 2) the $\phi_{0}$ angle estimation, localized at one intersection of the line with the trigonometric circle. Solution is determined after consideration of the interferometric coherence associated with the $\mathrm{HH}$ and/or $\mathrm{HH}+\mathrm{VV}$ channels, expected to be characterized by the most important groundto-volume power ratio, and with the HV channel, where the vegetation contribution is important; and 3) the interferometric coherence associated with the highest polarimetric phase center is assumed to be the "volume-only" one, i.e., without no-ground contribution. For numeric computation purposes, a lookup table (LUT) containing values of random volume interferometric coherences associated with all the possible mean extinction coefficients and heights is displayed in the complex plane and compared with the assumed pure volume interferometric coherence. The mean extinction coefficient and height which minimize the difference between the LUT and the assumed volume interferometric coherence measured in one polarimetric channel, and then the distance in the complex plane, are the inversion-solution couple. 
Dual-pol InSAR inversion is slightly different as the full set of polarization states associated with the transmit and receive antennas cannot be synthesized. Only a subset corresponding to the fixed transmit polarization can be explored. The line fit is then performed on this subset. One of these polarization states must correspond to the special case where $\mu=0$. This hypothesis must be done to solve the equation system constituted by (1) and (3) for the simultaneous extraction of the forest height and the mean extinction coefficient. At the L-band, this can be assumed to be the case for the HV channel [1], [2]. We expect this assumption to be even more valid at the X-band as the penetration is lower. The remaining high HV-phase-center position over the lowest tree density part of the forest indicates that the canopy contribution is still widely predominant even in the presence of gaps in the canopy in this polarimetric channel. Topography, which has been shown to create a strong ground return in HV [10]-[12], is not present in our data, and as there is also no understory under this managed forest, the assumption of no-ground contribution in the HV channel constitutes a realistic hypothesis. In case of significant relief, one solution would be to search for the linear receive polarization for which the return ground power is smaller and assumes that this is the volume-only contribution. Finally, note that this inversion procedure assumes no temporal decorrelation as this is a single pass.

Considering that $\mu_{\mathrm{HV}}=0$ and that both the ground and canopy contributions are received in the copolar channel $\left(\mu_{\mathrm{HH}}>0\right)$, the coherence equations are

$$
\begin{aligned}
\gamma_{\mathrm{HV}} & =e^{j \phi_{0}} \gamma_{v} \\
\gamma_{\mathrm{HH}} & =e^{j \phi_{0}}\left[\gamma_{v}+L_{\mathrm{HH}}\left(1-\gamma_{v}\right)\right] .
\end{aligned}
$$

The RVoG inversion is expected to be less accurate than for a Quad-Pol configuration because, in the Dual-Pol configuration, the linear regression in the complex plane relies on the subset of the full PolInSAR inversion and because, as a consequence, the polarimetric phase-center vertical dispersion is smaller.

\section{Pine Forest AnAlysis at X-BAnd}

The inversion relies on the assumption of no-ground contribution in the HV channel and of a ground/canopy contribution mix in the HH channel. Fig. 4 shows the corresponding polarimetric phase-center heights and points out a 6-m mean height difference over the whole pine forest corresponding to the vertical phase-center variation between these two polarimetric channels. However, the penetration depth of an isolated pine tree at X-band has been measured in the 0.5-1-m interval [6]. The observed discrepancy is suspected to result from the presence of gaps in the canopy in this sparse forest. Histograms in Fig. 4 have been shifted using the interferometric phase of a ground surface located in the close neighborhood of the forest to set the ground level at the origin of the diagram. The most populated bin is located around $10 \mathrm{~m}$ below the top of the canopy with, however, a noticeable percentage closer to the top, which corresponds to denser forest areas. Note that the histograms do not have a standard Gaussian shape, confirming the fact that the phase-center dispersion cannot be attributed to the sole phase accuracy (on the order of $2 \mathrm{~m}$ ), but must also result from either inhomogeneities of the forest structure or multiple-component scattering (surface + volume). More specifically, when looking at the histograms, we can distinguish two opposite behaviors between the $\mathrm{HH}$ and $\mathrm{HV}$ interferometric phases. The $\mathrm{HH}$ histogram is clearly nonsymmetrical, suggesting a two-component scattering mechanism (surface + volume) with a maximum corresponding to a low elevation, indicating a strong surface contribution. The forest height has been estimated with ground measurements to $18 \pm$ $1 \mathrm{~m}$ in mean. The HV histogram has a more symmetrical shape corresponding to a single volume contribution occurring around $4 \mathrm{~m}$ under the top canopy, with a scatter partially due to the forest height spatial heterogeneity. The VV interferometric phase estimation has shown that the associated phase center has an intermediate position between the $\mathrm{HH}$ and $\mathrm{HV}$ phase centers in the vertical direction. It means that $\mathrm{HH}$ and $\mathrm{HV}$ constitute the optimal polarization couple for the forest height inversion, as observed at L-band, i.e., the polarization pair presenting the largest height difference [5].

The black histogram shown in Fig. 4 is associated with the ground level, derived from the RVoG inversion procedure. The $\phi_{0}$ angle is directly estimated from the two polarimetric complex coherences and translated into height using the ambiguity height. That is why the estimated ground level follows a similar distribution to the two polarimetric phase-center heights (the standard deviations are equivalent). The 0 -m origin corresponds to a nearby ground surface level, and it appears that the black histogram is centered over this value in the absolute $2-\mathrm{m}$ interferometric height accuracy. The height difference computed between the HV phase center and the estimated ground level leads to a 4-m underestimation of the mean forest height $(18 \mathrm{~m})$. This confirms that the HV channel is dominated by the foliage contribution, which is in agreement with the assumption that the HV phase center is the "volume-only" one. Note, therefore, that if the assumption of no-ground contribution in the HV channel is not perfectly verified, this estimated 4-m penetration depth will still provide an upper bound, with the real penetration depth being smaller.

Fig. 5 shows the corresponding coherence histograms; the high values are expected in the single-pass case. These values are, however, significantly lower than the typical bare-surface coherences, measured on this data set at around 0.98 , because of the volume decorrelation induced by the penetration into the canopy.

At X-band, electromagnetic waves interact predominantly with leaf-scale objects [13] and present a sensitivity to the superficial topography of the forest canopy, i.e., the top-canopy height variations, contrary to lower frequencies, which penetrate deeper and interact with a thicker vegetation layer. The height of the volume scatterers, localized at the top of the canopy, can vary significantly over the estimation window. These height variations inside an averaging window are responsible for a scalar decorrelation of the "volume-only" contribution. Since this decorrelation only affects the canopy, the effect on the estimated interferometric coherence is similar to the temporal decorrelation one, which is due to the leaf movement induced by the wind, as described in [14]. 


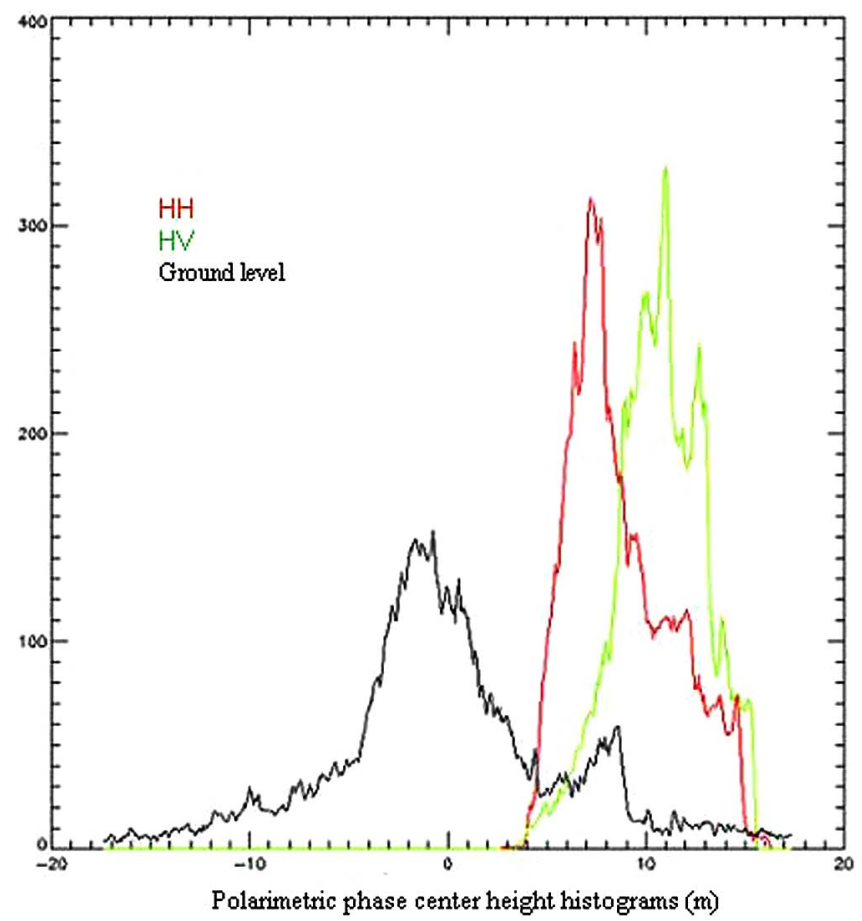

Fig. 4. Polarimetric-phase-center-height histograms. Red and green colors are, respectively, associated with the HH and HV channels. The black one corresponds to the ground level estimate.

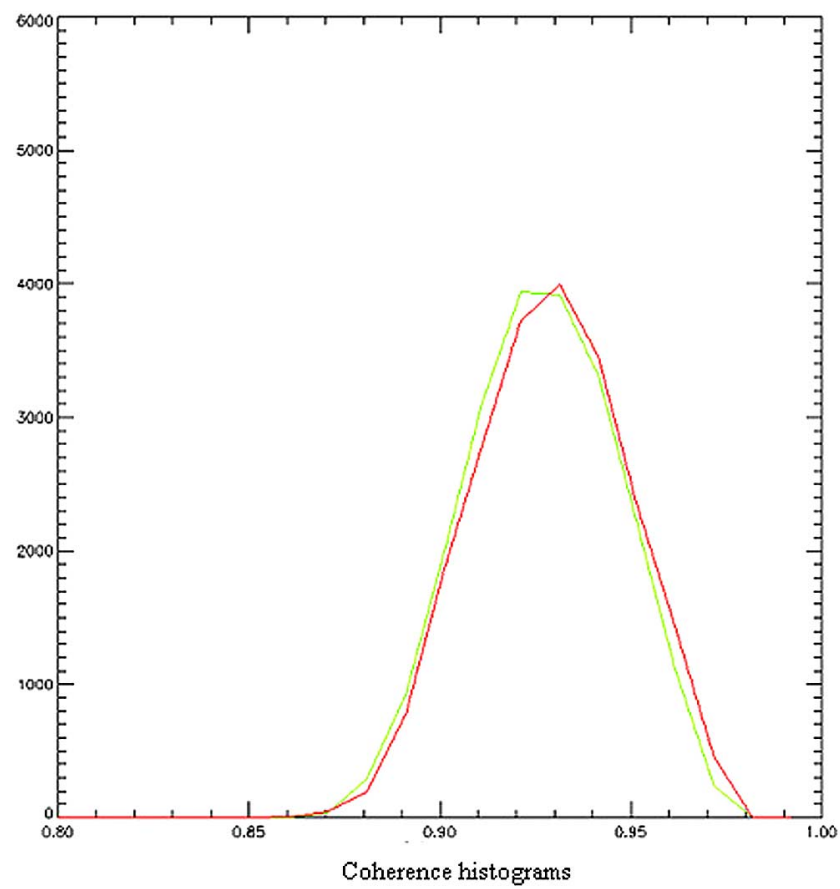

Fig. 5. Coherence histograms associated with (red) $\mathrm{HH}$ and (green) HV channels.

Let us consider (6), which is (1) rewritten after integration of this decorrelation coefficient $\alpha$ applied to the random-volume component only

$$
\gamma(\mathbf{w})=\left[\alpha \gamma_{v}+L(\mathbf{w})\left(1-\alpha \gamma_{v}\right)\right] e^{j \phi_{0}} .
$$

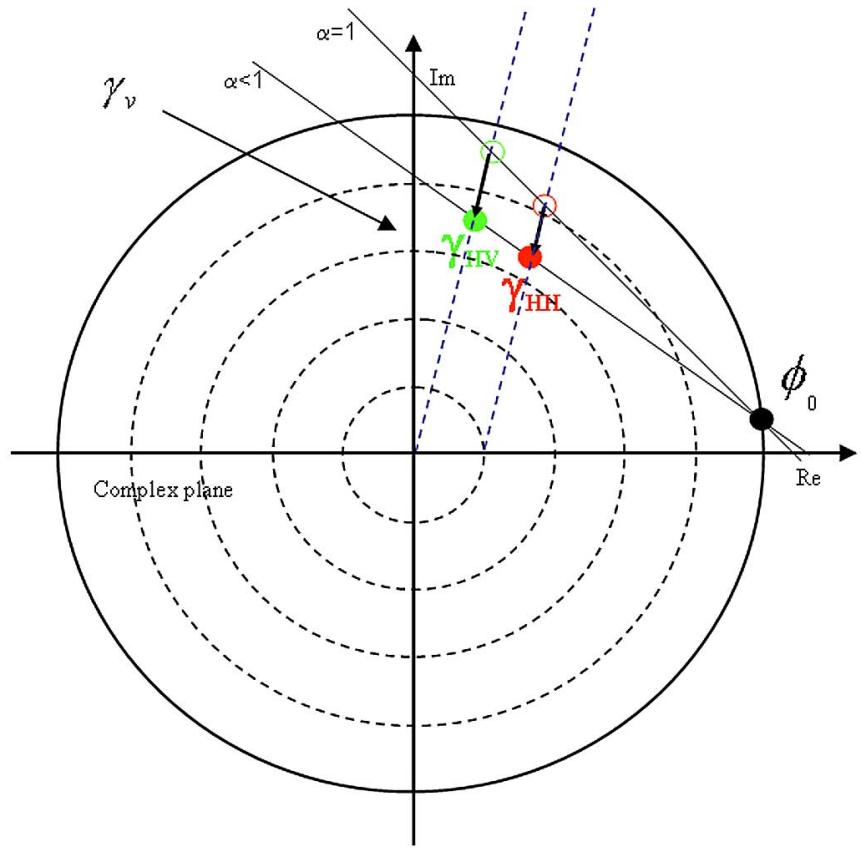

Fig. 6. Migration of the interferometric coherences $\gamma_{\mathrm{HH}}$ and $\gamma_{\mathrm{HV}}$ (assumed to be equal to $\gamma_{v}$ ) in the complex plane after decorrelation of the pure volume contribution. Circles correspond to theoretical interferometric coherences' positions without decorrelation, and the observed interferometric coherences are symbolized by plain circles.

As schematically shown in Fig. 6, the interferometric coherence of the volume $\gamma_{v}$, represented by $\gamma_{\mathrm{HV}}$, migrates toward the center of the trigonometric circle when the decorrelation increases, i.e., $\alpha$ decreases, and the interferometric coherences corresponding to the other polarizations follow the same linear direction, as shown in red for $\mathrm{HH}$ polarization. This decorrelation produces a decrease of the amplitude but does not affect the phase of the interferometric coherence $\gamma_{v}$, as shown in Fig. 6. Hence, the vertical location of the phase center corresponding to the "volume-only" contribution remains unchanged. Note that this decorrelation does not influence the estimation of the interferometric phase of the ground $\phi_{0}$ [14]. We can observe that the decorrelation of the pure volume contribution induces a coherence difference diminution between the polarizations, which explains the almost equal $\mathrm{HH}$ and $\mathrm{HV}$ coherences shown in Fig. 5.

Unfortunately, since the "volume-only" vertical position and coherence depend on both the mean extinction coefficient and the forest height, a scalar correction of $\left|\gamma_{v}\right|$ implies that the extinction coefficient must be fixed in order to estimate the forest height $h_{v}$ because, after correction, both parameters cannot be simultaneously estimated as in the "classical" $\mathrm{RVoG}$ inversion. The inversion procedure follows the following three steps.

1) Linear regression of the $\mathrm{HH}$ and $\mathrm{HV}$ interferometric coherences in the complex plane.

2) Ground-level estimation by the intersection of the line and the trigonometric circle, the intersection which allows the ground-level estimation being selected after 


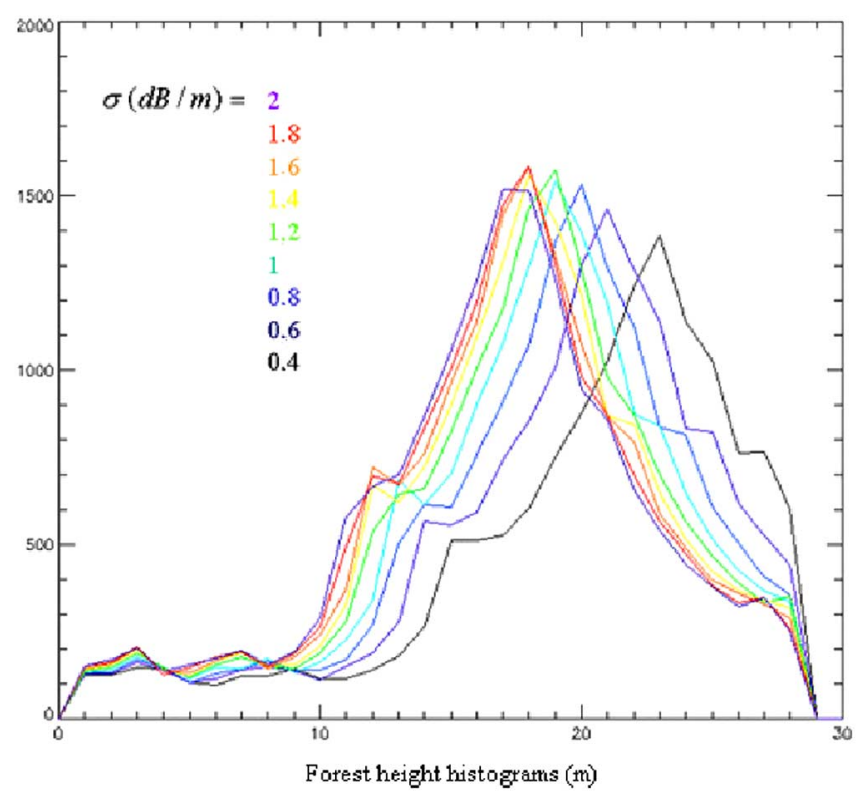

Fig. 7. Estimated forest height histograms corresponding to different mean extinction coefficients.

consideration of the polarimetric phase-center positions, as in a "classical" RVoG inversion procedure

$$
\left|\operatorname{Arg}\left\{\gamma_{\mathrm{HV}}\right\}-\phi_{0}\right| \geq\left|\operatorname{Arg}\left\{\gamma_{\mathrm{HH}}\right\}-\phi_{0}\right|
$$

It means that the $\mathrm{HH}$ phase center must be located closer to the ground than the HV phase center.

3) The validity domain of the "volume-only" interferometric coherence is located on the line joining the observed HV interferometric coherence and the complex-plane origin (blue dashed line crossing the green dot in Fig. 6). The volume interferometric coherence corresponding to a mean extinction coefficient value is computed for different heights. The intersection of the fixed extinction volume interferometric coherence with the line joining the complex-plane origin and the observed HV interferometric coherence correspond to the "volume-only" interferometric coherence before decorrelation (green circle in Fig. 6). The height associated with the fixed extinction interferometric coherence which corresponds to this intersection is the inversion solution. Note that HV coherence correction does not need any a priori information about the pure volume contribution decorrelation represented by the $\alpha$ parameter.

Fig. 7 shows the estimated forest height histograms associated with different mean extinction coefficients ranging from 0.4 to $2 \mathrm{~dB} / \mathrm{m}$. Since the accuracy of the forest height inversion is directly linked to the linear regression procedure and, consequently, to the ground-level estimation, these two parameters follow similar distributions (standard deviations are on the same order). The estimated height appears to be inversely proportional to the mean extinction coefficient: a given volume phase-center height should correspond to a high forest with a low extinction, where radar waves penetrate deeply, or to a

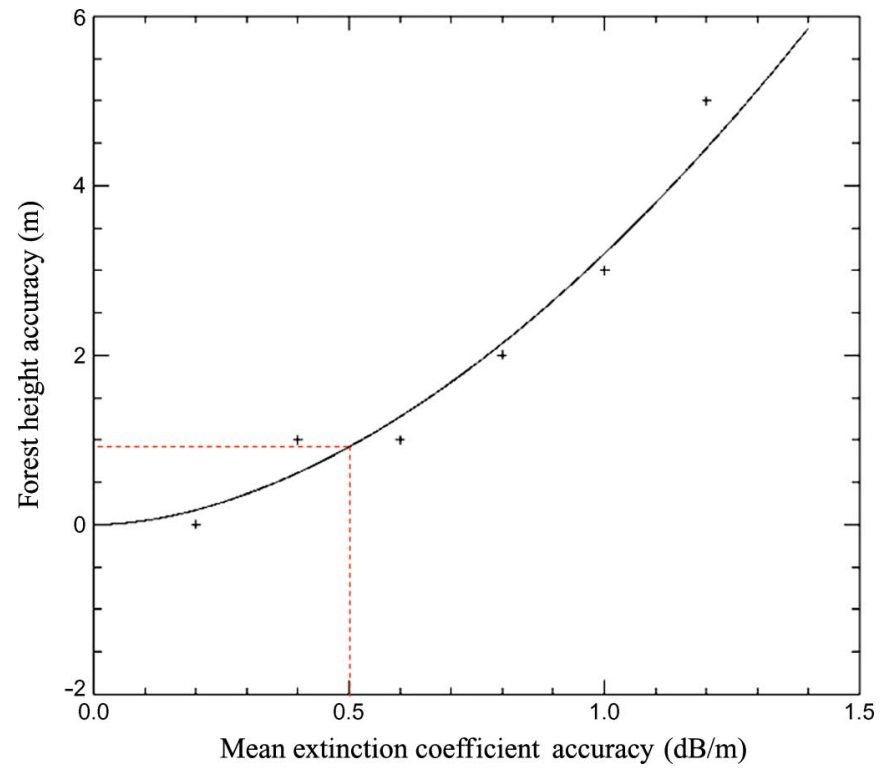

Fig. 8. Mean-height-estimation accuracy versus the mean extinction coefficient one.

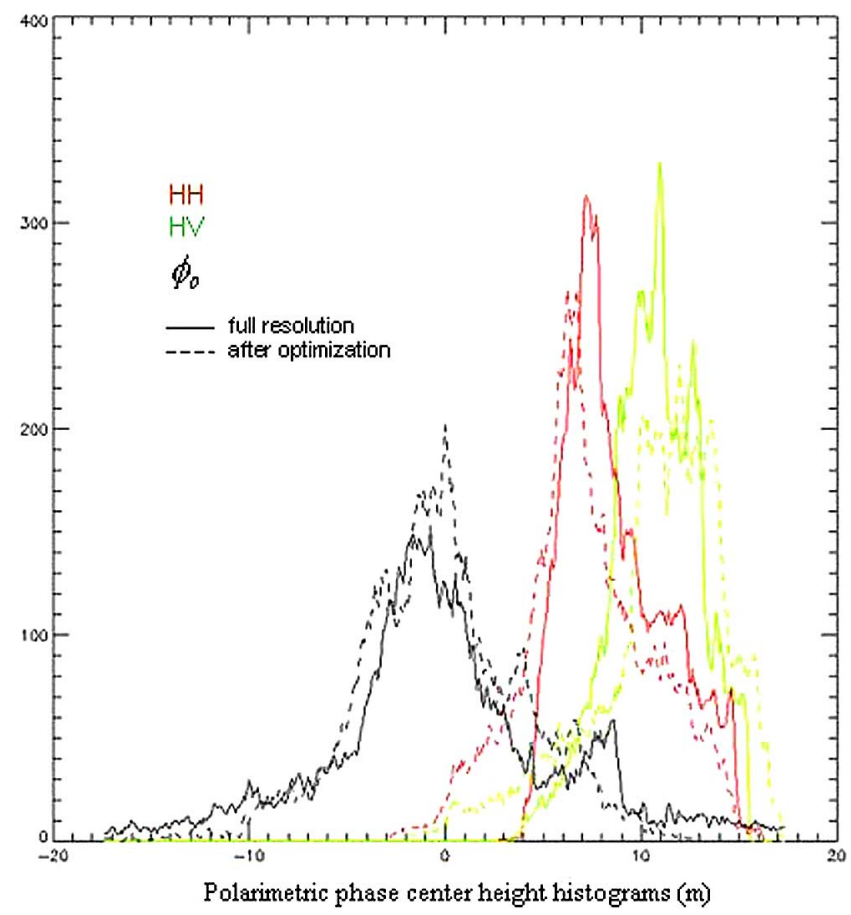

Fig. 9. Histograms of the phase-center height at (plain line) full resolution and (dotted line) after sublook optimization. Red and green colors are, respectively, associated with the $\mathrm{HH}$ and $\mathrm{HV}$ channels. The black one corresponds to the ground-level estimate.

smaller forest characterized by a higher extinction and then a lower penetration depth. The measured forest height during ground-truth campaigns, about $18 \mathrm{~m}$ in mean, is retrieved for high extinction values $(\sigma=1.6 \mathrm{~dB} / \mathrm{m})$, as expected at $\mathrm{X}$-band, because of a low penetration at this frequency [6]. The estimated extinction is expected to be valid over low-density forests because the tree density only affects the ground-tovolume power ratio and not the pure canopy interferometric coherence. Note that a large interval of extinction values gives 
a satisfying inversion, meaning that the mean extinction coefficient is not a sensitive parameter in this case because of its high level. The low dependence of the height inversion on the mean extinction coefficient is shown in Fig. 8: a variation of $0.5 \mathrm{~dB} / \mathrm{m}$ for the extinction values induces an estimated height error within the ground-measurement accuracy $(1 \mathrm{~m})$.

The decorrelation affecting the pure volume contribution, and then the correction taken into account in the inversion procedure, is now quantified. To do this, the theoretical volume coherence is compared to the coherence measured in the HV channel to estimate a decorrelation coefficient $\alpha$

$$
\alpha=\frac{\left|\gamma_{\mathrm{HV}}\right|}{\left|\gamma_{v\{h=18 \mathrm{~m}, \bar{\sigma}\}}\right|} .
$$

$\left|\gamma_{v\{h=18 \mathrm{~m}, \bar{\sigma}\}}\right|$ represents the interferometric coherence of an 18 -m height volume, characterized by a mean extinction coefficient comprised between 1 and $2 \mathrm{~dB} / \mathrm{m}$. Since the mean extinction coefficient does not influence strongly the volume interferometric coherence when it takes high values, it is averaged here. A mean decorrelation coefficient $\bar{\alpha}$ is then estimated around 0.92 . This mean value assumes the forest as a homogeneous medium to give a decorrelation order of the canopy topography, but this decorrelation coefficient can depend on the forest structure, which has not been investigated in this paper.

This last section has validated the RVoG hypotheses for a sparse pine forest at X-band. This forest can be modeled as a random volume characterized by a high mean extinction coefficient. The deep apparent penetration in the $\mathrm{HH}$ channel and the distinct height distributions of the polarimetric phase centers may be explained by the presence of gaps in the canopy. The gaps allow ground-backscatter reception in the HH channel, whereas the HV channel remains mainly dominated by the canopy contribution. In the next section, a TFA is performed to use the natural azimuth look-angle variation in SAR data for a better separation of the polarimetric phase centers and for an $\mathrm{RVoG}$ inversion performance improvement.

\section{TIME-FREQUENCY Optimization}

In this section, an azimuth sublook analysis is performed to remove the ground contribution contained in the $\mathrm{HV}$ channel to better suit the RVoG assumptions.

In SAR, the azimuth resolution is obtained through a signal processing technique called azimuth compression. It relies on the fact that the relative movement between the platform and the target induces a varying Doppler frequency $f_{d}$ along the integration acquisition time [15] during which the scatterer is seen with a varying observation angle $\psi$. The resulting Doppler bandwidth is directly proportional to the observation angle variation interval and is responsible for the azimuth resolution, as can be seen in the following equation, linking the observation angle variation interval $\Delta \psi$ and the azimuth spatial resolution $\delta_{\mathrm{az}}$

$$
\Delta \psi=\frac{\lambda}{2 \delta_{\mathrm{az}}}
$$

where $\lambda$ is the radar wavelength.
A given Doppler frequency corresponds to an observation angle. Therefore, the filtering of image spectra in the azimuth direction is equivalent to selecting the received information corresponding to a given observation angle

$$
\psi=\arcsin \left(\frac{f_{d} \lambda}{2 v_{\mathrm{p}}}\right)
$$

where $v_{\mathrm{p}}$ is the platform velocity.

Note that the reduction of the Doppler bandwidth corresponds to a decrease of the observation angle interval and results in a coarsening of the azimuth resolution (8). The look-angle-dependent information is directly related to the geometrical properties of the illuminated scatterers [16], [17]. In the present analysis, we are interested in separating the contributions coming from different apparent look observation angles $\psi$. Fast Fourier transform filtering of the SAR image spectra in the azimuth direction is done to form five sublook images of equivalent resolutions with $50 \%$ overlap, whereas the full resolution is kept in the range direction. The complex interferometric coherences for the HH and HV channels over the five sublook images are computed over a sliding window as in the previous section, but the window size is adapted in order to keep a sufficient number of looks to avoid any estimation bias [18]. Because of a small observation angle variation $(\Delta \psi \simeq$ $1^{\circ}$ ), only three of these five sublooks are independent. Before investigating the pine forest, bare-soil surfaces are considered and analyzed. The interferometric behavior is fairly constant from one sublook to the next over these surfaces, displaying no specific trend and guaranteeing an artifact-free system. Over vegetation, a significant spread in phase centers and interferometric coherences across the different sublooks is observed. A physical interpretation can be proposed. The 30 incidence-angle geometries have the following two main consequences: 1) even small gaps in the canopy will create a direct ground visibility, i.e., the electromagnetic wave can travel freely all the way to the ground and 2) the scattering from the gaps will result in contributions from the whole vertical structure.

The TFA optimization consists of selecting for each averaging window the sublook which maximizes the height difference between the $\mathrm{HH}$ and $\mathrm{HV}$ phase centers. The goal of this optimization is to increase the ground-to-volume power-ratio range between the two polarimetric channels. Fig. 9 shows the resulting height histograms associated with both polarizations. After the optimization, the HH histogram is shifted toward a lower elevation compared to the full-resolution one, whereas the HV histogram follows an opposite behavior. The separation between the two polarimetric phase centers is increased by about $3 \mathrm{~m}$ in mean. At the same time, the filtering of the contributions leads to a coherence enhancement (Fig. 10). The height histograms are observed to be narrower, providing a better estimate of the ground level. The height difference between the $\mathrm{HV}$ phase center and the estimated ground level is observed to be around $16 \mathrm{~m}$, leading to a $2-\mathrm{m}$ penetration-depth estimation at X-band based on forest height ground measurements, which is more consistent with previous observations [6]. 


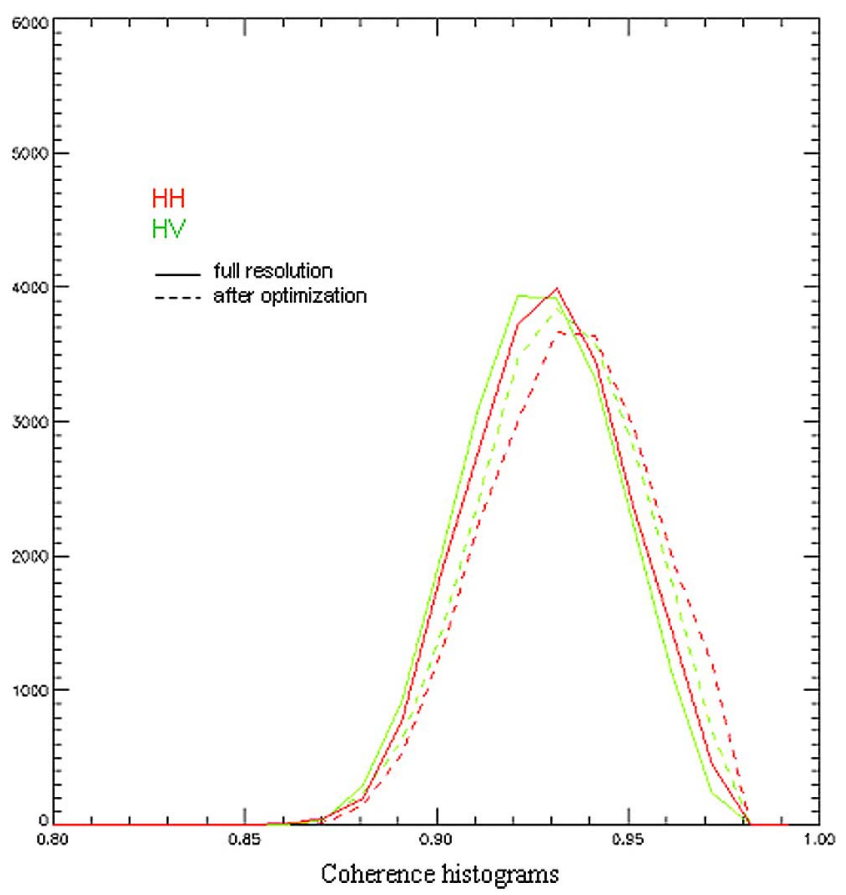

Fig. 10. Coherence histograms (plain line) in the full-resolution case and (dotted line) in the sublook optimization one. Red and green colors are, respectively, associated with the $\mathrm{HH}$ and $\mathrm{HV}$ channels.

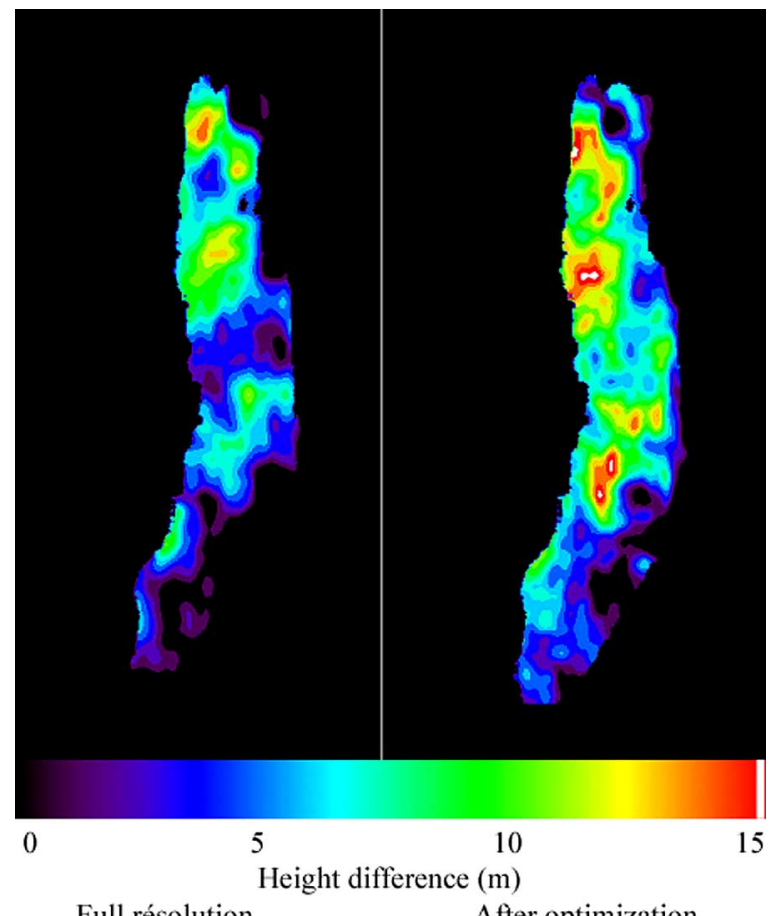

Full résolution

After optimization

Fig. 11. Height-difference map between the HV phase center and the $\mathrm{HH}$ one. The left map corresponds to the full-resolution data, and the right one is associated with the sublook-optimized one.

In Fig. 11, the phase difference between HV and HH is represented over the pine forest extend. The left part of Fig. 11 shows the initial phase difference, computed over the full-resolution data set. The right map represents the phase difference obtained

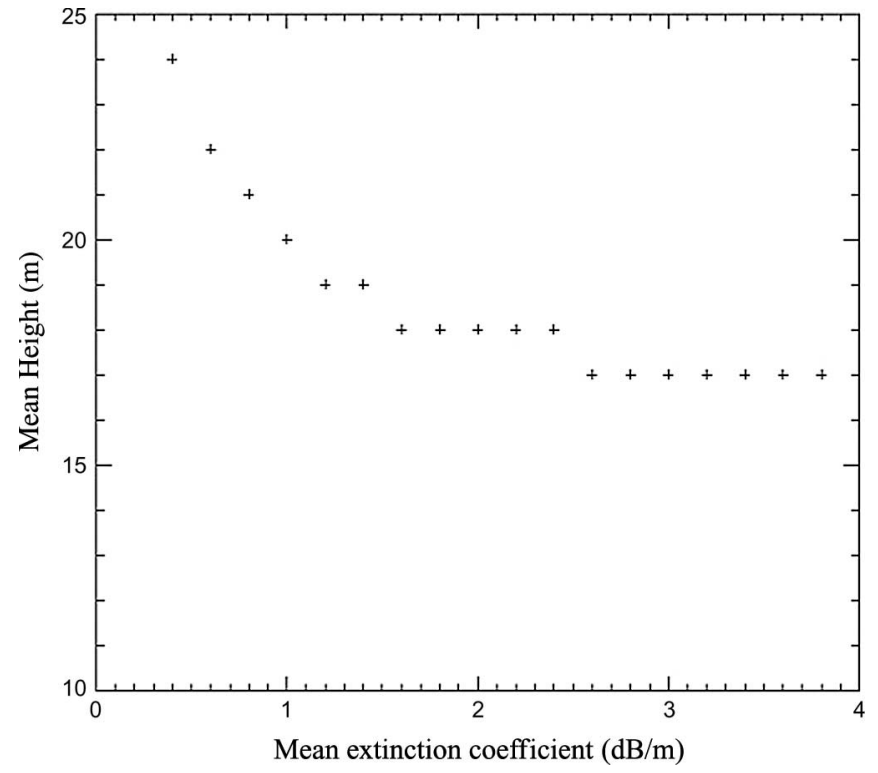

Fig. 12. Mean-estimated forest height versus mean extinction coefficient after the time-frequency optimization.

after maximization over the sublook images. The left side of Fig. 11 shows that the most important polarimetric phase-center separation is found in the lowest tree density part of the forest (located at the top part of both figures), i.e., containing the most gaps in the canopy. The ground component in the $\mathrm{HH}$ backscatter is more important than in the denser area, whereas the canopy remains highly predominant in the HV channel over the whole forest. Then, the phase-center height difference between the $\mathrm{HV}$ and $\mathrm{HH}$ polarizations tends to increase in the low-density area because of $\mathrm{HH}$ scattering center-height diminution. Note that, in the low-density areas, the HV channel is still dominated by canopy contribution. On some pixels, sublook decomposition allows an almost total separation of the ground and volume components, varying the ground-to-volume power ratio with polarization over its full range. In this sense, the time-frequency approach compensates the gap effect in the crosspolar channel and increases it in the copolar channel. After sublook maximization, the $\phi_{0}$ histogram becomes slightly narrower (Fig. 9), and the ground-level estimation is then more robust. The estimation of the forest height also becomes more precise because of the elongation of the region of coherence in the complex plane. Furthermore, note that, in this X-band context, since the HV phase center is closer to the top of the canopy (more representative of the "volumeonly" contribution compared to the full-resolution case), the solution mean extinction coefficient allowing forest height retrieval then becomes higher $(\sigma=2 \mathrm{~dB} / \mathrm{m})$, as shown in Fig. 12 . We can observe that the curve describing the estimated height as a function of the mean extinction coefficient reaches an asymptotic value for large extinctions, equal to the HV phasecenter height, reflecting a null penetration. Since the penetration depth decreases until $2 \mathrm{~m}$ after time-frequency optimization, the measure of the HV phase-center height after ground-level determination only provides a small underestimation of the forest height. 


\section{CONCLUSION}

This paper has presented the first results of the investigation of X-band single-pass PolInSAR data acquired over a pine forest using only $\mathrm{HH}$ and $\mathrm{HV}$ polarizations. A significant height difference between the two polarimetric phase centers has been first observed, linked to the penetration into the canopy and to the different ground-to-volume power ratios. Previous work has shown a 1-m penetration depth in an isolated pine tree at $\mathrm{X}$-band [6]. The observed deeper apparent penetration of radar waves in the vegetation layer in the two polarimetric channels can be attributed to the presence of gaps in the canopy. This effect is enhanced by the steep geometry for which even small gaps create significant ground visibility.

Using these two polarizations, the forest parameter extraction is conducted using the RVoG model assumptions [1], [2]. The assumption of no-ground contribution in the HV channel, attested by a high phase-center position over the whole forest, is done to estimate the random-volume interferometric coherence. The effect of the "roughness" of the canopy, i.e., variation of the canopy height in an estimation window, has been observed on our X-band data, inducing a decorrelation of the pure canopy contribution. An inversion procedure correcting this effect without no a priori information concerning its amount has been employed to estimate the forest height by using assumption only on the mean extinction coefficient. As expected, the solution mean extinction coefficient allowing the correct forest height retrieval (in accordance with the ground measurements) is high, which is consistent with the limited penetration depth at X-band. In consequence, the RVoG model inversion is not very sensitive to this parameter, and the forest height can be retrieved accurately for a large interval of the assumed extinction coefficients. This limited influence of the mean extinction coefficient makes forest height extraction with the adapted RVoG model robust at X-band. The first penetration depth of the X-band waves in the canopy is estimated to be around $4 \mathrm{~m}$ by computing the difference of the forest height, obtained by ground measurements, and the HV phase-center elevation.

Then, to improve the separation of the soil and canopy contributions and to increase the accuracy of the RVoG inversion, a TFA was conducted. SAR images' frequency spectra are decomposed in different sublooks in the azimuth direction in order to select contributions coming from different look angles. On each sliding window, the sublook that provides the best vertical separation of the $\mathrm{HH}$ and $\mathrm{HV}$ polarimetric phase centers is selected. The soil-contribution reduction in the HV channel allows obtaining more accurately the "volume-only" contribution. The time-frequency approach is then used to compensate the gap effect in the HV channel and to enhance it in the $\mathrm{HH}$ channel, where the ground backscatter is increased. The height difference between the two polarimetric phase centers is increased by $3 \mathrm{~m}$ over the whole forest, enhancing the forest height inversion performance using the adapted RVoG model. After the time-frequency optimization, the HV phase center migrates toward the top of the canopy, and the estimated mean extinction coefficient, allowing the correct forest height retrieval, becomes higher. The penetration depth of X-band in the forest canopy is then estimated more accurately to be $2 \mathrm{~m}$, reaching the system accuracy, which is in better accordance with [6].

To measure tree heights, airborne techniques use the assumptions of no X-band penetration in the canopy [19]. This paper has shown that when the forest density decreases, the apparent penetration depth difference between the polarimetric channels increases due to gaps in the canopy, which makes the PolInSAR inversion possible. Note that by using fully polarimetric interferometric data ( $\mathrm{HH}, \mathrm{HV}, \mathrm{VH}$, and VV channels), inversion performance is expected to be improved compared to the Dual-Pol configurations.

With the launch of the TandemX-SAR scheduled in 2008 [20], comparable scenes will be available for different forest contexts, since altimetric accuracy and incidence angle are similar. Nevertheless, only a tandem mission should provide equivalent data, without temporal decorrelation. However, compared to lower frequencies, the performance of the vegetation analysis with the PolInSAR technique will be more densitydependent at X-band. The reception of the ground component at high frequency is conditioned by the presence of gaps in the canopy, whereas penetration always occurs in the forest at lower frequencies. Consequently, the PolInSAR inversion at X-band needs to be evaluated now on denser forests. The influence of tree density on the PolInSAR signal and on inversion needs to be quantified for a complete evaluation of the $\mathrm{X}$-band potential for forest height estimation using single-pass PolInSAR data. The use of this frequency also provides higher spatial resolutions, and since the interaction concerns leaf-scale objects, it allows investigation of textural aspects of vegetation.

\section{ACKNOWLEDGMENT}

The authors would like to thank the RAMSES team for the great data set, the RITAS team for its motivation and enthusiasm in putting together the project and collecting the ground truth (particularly the INRA team who hosted the experiment), the French Armament Procurement Agency for authorizing the authors to fly two science lines during their campaign, and the French Flight Test Center for the aircraft platform operation during the acquisitions. The authors would also like to thank particularly D. Heuzé (Office National d'Études et de Recherches Aérospatiales) for the help in the collection of the ground measurements and P. Paillou for the helpful discussions.

\section{REFERENCES}

[1] K. P. Papathanassiou and S. R. Cloude, "Single-baseline polarimetric SAR interferometry," IEEE Trans. Geosci. Remote Sens., vol. 39, no. 11, pp. 2352-2363, Nov. 2001.

[2] S. R. Cloude and K. P. Papathanassiou, "A 3-stages inversion process for polarimetric SAR interferometry," Proc. Inst. Electr. Eng.-Radar Sonar Navig., vol. 150, no. 3, pp. 125-134, Jun. 2003.

[3] T. Mette et al., "Performance of forest biomass estimation from PolInSAR and forest allometry over temperate forests," in Proc. POLInSAR, Frascati, Italy, 2005.

[4] P. Dubois-Fernandez et al., "The ONERA RAMSES SAR: Status in 2004," in Proc. RADAR Symp., Toulouse, France, Oct. 2004.

[5] K. P. Papathanassiou and S. R. Cloude, "Forest height estimation using Dual-Pol InSAR configurations," in Proc. EUSAR, Ulm, Germany, May 2004, pp. 93-96. 
[6] E. Mougin, A. Lopez, M. A. Karam, and A. K. Fung, "Effect of tree structure on X-band microwave signature of conifers," IEEE Trans. Geosci. Remote Sens., vol. 31, no. 3, pp. 370-381, May 1993.

[7] P. Dubois-Fernandez, X. Dupuis, and F. Garestier, "PolInSAR calibration of deramp-on-receive system: Example with the RAMSES airborne system," Can. J. Remote Sens., vol. 31, no. 1, pp. 112-121, 2005.

[8] J. I. H. Askne, P. B. G. Dammert, L. M. H. Ulander, and G. Smith, "C-band repeat-pass interferometric SAR observations of the forest," IEEE Trans. Geosci. Remote Sens., vol. 35, no. 1, pp. 25-35, Jan. 1997.

[9] R. N. Treuhaft, S. Madsen, M. Moghaddam, and J. J. van Zyl, "Vegetation characteristics and underlying topography from interferometric data," Radio Sci., vol. 31, pp. 1449-1495, Dec. 1996.

[10] D. L. Schuler, J. S. Lee, and G. De Grandi, "Measurement of topography using polarimetric SAR images," IEEE Trans. Geosci. Remote Sens., vol. 34, no. 5, pp. 1266-1277, Sep. 1996.

[11] J. S. Lee, D. L. Schuler, and T. L. Ainsworth, "Polarimetric SAR data compensation for terrain azimuth slope variation," IEEE Trans. Geosci. Remote Sens., vol. 38, no. 5, pp. 2153-2163, Sep. 2000.

[12] J. J. van Zyl, "The effect of topography on radar scattering from vegetated areas," IEEE Trans. Geosci. Remote Sens., vol. 31, no. 1, pp. 153-160, Jan. 1993.

[13] F. Garestier, P. Dubois-Fernandez, X. Dupuis, P. Paillou, and I. Hajnsek, "PolInSAR analysis of X-band data over vegetated and urban areas," IEEE Trans. Geosci. Remote Sens., vol. 44, no. 2, pp. 356-364, Feb. 2006.

[14] K. P. Papathanassiou and S. R. Cloude, "The effect of temporal decorrelation on the inversion of forest parameters from Pol-InSAR data," in Proc. IGARSS, Toulouse, France, Jul. 2003, pp. 1429-1431.

[15] J. C. Curlander and R. N. McDonough, Synthetic Aperture Radar: Systems and Signal Processing, ser. Wiley Series in Remote Sensing. New York: Wiley, 1991.

[16] J. C. Souyris, C. Henry, and F. Adragna, "On the use of complex SAR image spectral analysis for target detection: Assessment of polarimetry," IEEE Trans. Geosci. Remote Sens., vol. 41, no. 12, pp. 2725-2734, Dec. 2003.

[17] L. Ferro-Famil, A. Reigber, E. Pottier, and W.-M. Boerner, "Scene characterization using subaperture polarimetric SAR data," IEEE Trans. Geosci. Remote Sens., vol. 41, no. 10, pp. 2264-2276, Oct. 2003.

[18] R. Touzi et al., "Coherence estimation for SAR imagery," IEEE Trans. Geosci. Remote Sens., vol. 37, no. 1, pp. 135-149, Jan. 1999.

[19] S. Hensley et al., "First P-band results using the GeoSAR mapping system," in Proc. IGARSS, Sydney, Australia, 2001, vol. 1, pp. 126-128.

[20] I. Hajnsek and A. Moreira, "TanDEM-X: Mission and science exploration," in Proc. POLInSAR, Frascati, Italy, Jan. 2007.

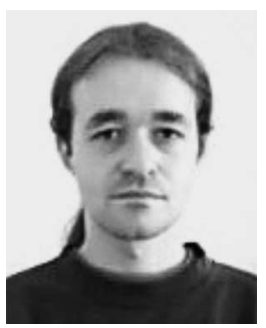

Franck Garestier received the M.Sc. degree in geology-geophysics from the Université Paul Sabatier, Toulouse, France, in 2002, and the Ph.D. degree in SAR remote sensing from the Université de Bordeaux, Bordeaux, France, in 2006.

From 2003 to 2006, he was with the Office National d'Études et de Recherches Aérospatiales, Salon-de-Provence, France, to evaluate the polarimetric SAR interferometry (PolInSAR) technique potential for vegetation physical-parameter inversion. He is currently with the Centre d'Etudes Spatiales de la Biosphère, Toulouse, France, working on forestry applications using low-frequency SAR data. His research interests concern vegetation physical-parameter estimation using advanced SAR techniques (polarimetry, interferometry, and PolInSAR), including data calibration, vegetation modeling, inversion algorithms development, and spaceborne condition investigation.

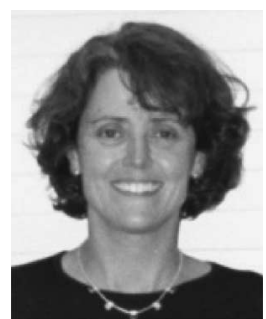

Pascale C. Dubois-Fernandez (M'04-A'04SM'04) received the Dipl.Ing. degree from the Ecole Nationale Supérieure d'Ingénieur de Constructions Aéronautiques, Toulouse, France, in 1983, and the M.S. and Eng. degrees from the California Institute of Technology, Pasadena, CA, in 1984 and 1986, respectively.

She was with the Radar Science and Technology Group at the Jet Propulsion Laboratory, Pasadena, where she stayed for ten years, participating in numerous programs like Magellan, Airborne SAR, and Spaceborne Imaging Radar-C. She then moved back to France, where she worked on cartographic applications of satellite data. Since 2000, she has been with the Department of Electromagnetism and Radar/RIM, Office National d'Études et de Recherches Aérospatiales (ONERA), Salon-de-Provence, France, the French Aeronautics and Aerospace Research Institute, where she has been involved in the ONERA SAR airborne platform, RAMSES, developing science applications.

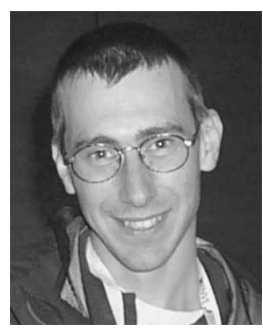

Konstantinos Panagiotis Papathanassiou (M'02SM'03) received the Dipl.Ing. (with honors) and Ph.D. degrees (with honors) from the Technical University of Graz, Graz, Austria, in 1994 and 1999, respectively.

From 1992 to 1994, he was with the Institute for Digital Image Processing (DIBAG), Joanneum Research, Graz. Between 1995 and 1999, he was with the Microwaves and Radar Institute (HR), German Aerospace Center (DLR), Wessling, Germany. From 1999 to 2000, he was an EU Postdoctoral Fellow with applied electromagnetics (AEL), St. Andrews, Scotland. Since October 2000, he has again been with the Microwaves and Radar Institute (HR), DLR, where he is a Senior Scientist within the Polarimetric SAR Interferometry (PolInSAR) Group working on PolInSAR and the development of inversion algorithms for physical parameters from SAR data. His main research interests are in polarimetric and interferometric processing and calibration techniques, polarimetric SAR interferometry, and quantitative parameter estimation from SAR data. He has more than 100 publications in international journals, conferences, and workshops.

Dr. Papathanassiou was awarded with the IEEE Geoscience and Remote Sensing Society International Geoscience and Remote Sensing Symposium Prize Paper Award in 1998, the Best Paper Award of the European SAR Conference in 2002, and the DLR Science Award in 2002. 